\title{
Assessment of the tsunami hazard on Moroccan coasts using numerical modeling
}

\author{
Meriem Amine ${ }^{1}$, Latifa Ouadif ${ }^{1}$, Khadija Baba $^{2} \&$ Lahcen Bahi $^{1}$ \\ ${ }^{1}$ Laboratory of applied Geophysics, Geotechnics, Geology of the Engineer and Impact on the Environment (L3GIE), \\ Mohammadia School of Engineers, Mohamed V University, Rabat, Morocco. \\ ${ }^{2}$ Laboratory of civil engineering and environment (GCE), High School of Technology-Sale, Mohamed V University, \\ Rabat, Morocco.
}

\begin{abstract}
Caused by earthquakes in the marine environment, tsunamis are often of particular violence. Pests, their damage far exceeds those caused by earthquakes earthquakes. In Morocco, seismic sensors in the national network often detect earthquakes in the marine environment. However, specialists minimize their threat given the peculiarity of the Moroccan ocean zone. The latter is characterized by the importance of the length of the faults and the depth of the seismic focus.

This work can be considered as a first approach to the study and understanding tsunamis. Although Morocco has known since historical times, earthquakes and tsunamis along its coasts.

This preliminary study is mainly intended to show the contribution of the numerical simulation of tsunamis, with a concrete application of the 1755 Tsunami of Lisbon based on the four source zones involving the five potentially tsunamigenic faults that are generally mentioned in the literature for this major event.
\end{abstract}

\section{Introduction}

Le tsunami est une catastrophe naturelle parmi les plus destructrices de l'histoire, il est perçu comme une série de vagues océaniques destructrices déferlantes de très grande hauteur et ayant été provoquée par de brutales perturbations de la couche d'eau de l'océan, causant de nombreux dégâts.

La majorité des tsunamis est générée par des séismes se produisant en domaine maritime, mais seuls les séismes de forte magnitude et de faible profondeur hypocentrale génèrent des tsunamis, en effet le séisme doit générer une déformation suffisante du fond de l'océan (de l'ordre de quelques mètres). C'est pour cette raison que la plupart des séismes générant des tsunamis ont lieu le long de grandes zones de subduction. À l'approche des côtes, l'amplitude augmente au détriment de la vitesse, qui baisse avec la profondeur d'eau. En conséquence le train d'onde sera comprimé spatialement, et son amplitude deviendra plus importante. Dans les cas les plus forts, l'onde peut atteindre quelques dizaines de mètres de hauteur et inonder sur des distances de plusieurs kilomètres. Lors de l'inondation, lorsque l'onde pénètre dans les terres, les dégâts peuvent être considérables: arbres coupés, infrastructures détruites, déplacement de masses importantes (bateaux, conteneurs,...). Les caractéristiques des ondes tsunamis lors de l'inondation dépendent de la morphologie des terrains.

Dans la région de l'Atlantique Nord-Est, les pays les plus exposés aux tsunamis sont le Maroc, le Portugal et
l'Espagne [1; 2; 3] (Figure 1). Dans ces pays, les structures côtières et de défense de la mer ne sont pas conçues pour résister à l'impact du tsunami et ne servent que des brise-lames qui protègent les marinas et les installations portuaires des vagues de l'océan.

Au Maroc, l'aléa tsunami peut être considéré comme étant à faible probabilité, mais à forte gravité du fait de l'importance des enjeux présents, et surtout à venir, sur la bande littorale.

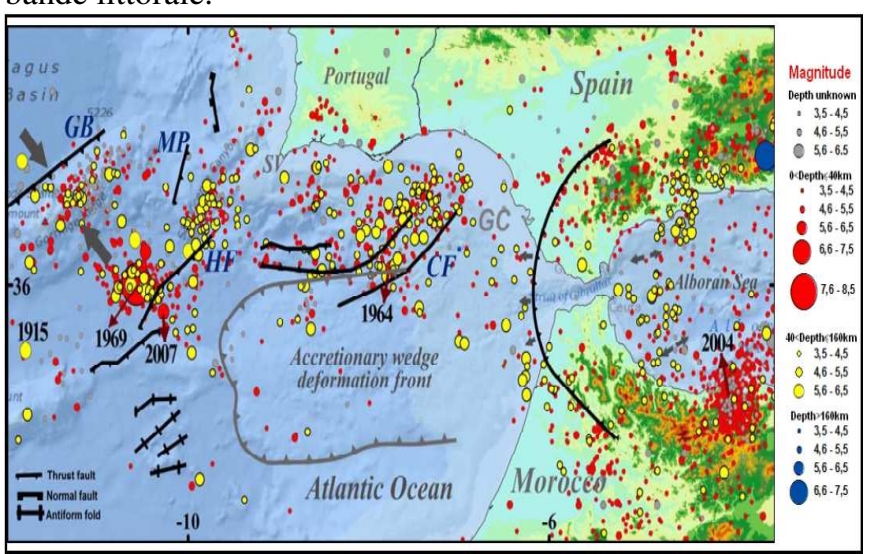

Fig. 1. Carte de sismicité de la zone Açores-Gibraltar. Failles majeures (de Gutsher et al., 2009 et Rosas et al., 2012) et

distribution d'hypocentres sismiques de $\mathrm{M} \geq 3,5$ pendant la période 1901-2010.

Dans cette étude nous considérons cinq scénarios tsunamigéniques, tous d'origine sismique. Ces scénarios, de grandeur allant de 8,0 à 8,6 , représentent les principaux défauts de tremblement de terre actifs dans l'océan atlantique qui peuvent déclencher de gros 
tsunamis dans la région. La valeur inférieure $(\mathrm{Mw}=8.0)$ est compatible avec l'événement du 28 février 1969 [1; $4]$, tandis que les grandeurs supérieures $(\mathrm{Mw}=8.2-8.6)$ sont considérées comme représentant l'événement du $1^{\mathrm{er}}$ novembre 1755 [1]. Nous effectuons une modélisation numérique de la propagation du tsunami pour tous les scénarios considérés et l'inondation pour le scénario qui a causé le pire impact dans la zone étudiée.

Les résultats prévus montrent différents niveaux d'impact que les différentes conditions de l'onde de tsunami pourraient générer dans la région. L'impact dramatique du tsunami dans la région montre la nécessité de nouvelles normes de tsunami non seulement pour les structures de défense de la mer, mais aussi pour les habitations côtières et les maisons afin de fournir une évacuation potentielle sur place.

La conception et la construction d'installations côtières capables d'arrêter l'inondation du tsunami dans les zones côtières à risque demeurent l'une des mesures recommandées pour atténuer les risques liés aux tsunamis. D'autre part, les normes de construction des défenses maritimes doivent répondre aux besoins de sécurité des zones vulnérables, qui pourraient découler des estimations et des prévisions d'impact et de dégâts.

L'inondation du tsunami et la propagation des ondes terrestres dépendent de manière critique de la qualité du terrain numérique et des modèles numériques de propagation du tsunami. Le modèle numérique du terrain doit inclure une description complète de la bathymétrie, de l'infrastructure topographique et côtière [5].

\section{Evaluation du risque tsunami sur le littoral marocain}

Le Maroc, par son contexte géologique particulier et sa proximité de la limite de plaque Nubie-Eurasie (NEPB) est le littoral ouest africain qui est le plus exposé aux tsunamis induits par les séismes [6] (figure1). Les tremblements de terre sous-marins les plus graves ressenties au Maroc ont été ceux générés en mer le long de la côte atlantique $[7 ; 8 ; 9 ; 10]$. Certains de ces événements étaient tsunamigène, comme ce fut le cas de l'événement du $1^{\text {er }}$ Novembre 1755, qui était certainement le tsunami le plus dévastateur jamais enregistré au Maroc. Ses vagues ont ravagé les côtes ibériques et atlantique marocaine, avec des hauteurs qui ont atteint 5-15 m et ont été observées à travers l'Atlantique dans les Antilles [11], mais il persiste encore de nombreuses incertitudes sur la source exacte du séisme et ses effets sur les côtes, en particulier marocaines. Il s'agit du seul tsunami connu en Atlantique nord et demeure en cela un événement de référence pour établir des scénarios de risque sur cet espace.

\section{Modélisation de l'aléa tsunami sur les côtes marocaines}

La modélisation numérique, développée depuis les années 1960, consiste à construire un ensemble de fonctions mathématiques décrivant le phénomène. Elle repose sur la programmation de modèles théoriques ou mathématiques (intégration des équations du mouvement, d'équations différentielles, etc...) qui sont adaptés aux moyens numériques. En modifiant les variables de départ, on peut ainsi prédire les modifications du système physique.

Dans le cas des études de tsunamis, la modélisation numérique permet d'évaluer l'aléa et de proposer, sur la base d'analyses sismotectoniques, des scénarios crédibles dans des zones où les données historiques sont incomplètes [12].

La modélisation de tsunami consiste à reproduire des événements passés selon une approche déterministe ou en imaginer de nouveaux. Elle comprend trois phases distinctes: 1) la génération du tsunami, 2) la propagation au large et 3) l'interaction des ondes avec le plateau continental et l'inondation de la côte.

\subsection{Code de simulation}

Les connaissances sur les tsunamis historiques ayant affecté le Maroc sont insuffisantes pour estimer l'aléa. Par conséquent, la modélisation numérique est le seul moyen de déterminer, au moins approximativement, les zones qui seront inondées, les hauteurs d'eau et les temps d'arrivée d'un tsunami d'origine sismique. Afin de sensibiliser les décideurs sur la réalité de ce risque, l'événement de référence retenu pour ce modèle est le tsunami du $\quad 1^{\text {er }}$ novembre 1755. Les simulations numériques du tsunami effectuées dans le cadre de ce travail ont été réalisées avec le code SWAN (Simulating WAves Nearshore) sous le logiciel Mirone. SWAN est un modèle de vague de troisième génération, qui calcule des ondes aléatoires, à crête courte, générées par le vent dans les régions côtières et les eaux intérieures.

La rupture sismique est censée être instantanée et le déplacement du fond marin est calculé en utilisant la théorie du demi-espace élastique [13], mise en œuvre dans le code MIRONE. Le déplacement vertical du fond de la mer est ensuite transféré à la surface libre de l'océan, selon l'hypothèse que les deux déformations, du fond de la mer et de la surface de l'océan, seraient égales. Ce modèle consiste dans un premier temps à calculer la déformation initiale associée à une rupture sismique en faisant appel aux algorithmes développés par Okada (1985) qui correspondent à la méthode couramment utilisée par les différents modèles (la théorie demi-espace élastique) [14]. Ces algorithmes permettent de calculer la déformation du plancher océanique à partir des caractéristiques de la faille à l'origine du séisme. Le tsunami initial est ensuite déduit en admettant que l'intégralité de cette déformation est instantanément transmise à la surface de l'eau.

\subsection{Scénarios tsunamigènes}

La source exacte du tremblement de terre du $1^{\text {er }}$ Novembre 1755 demeure encore à ce jour incertaine [15]. Quatre zones sources impliquant cinq failles potentiellement tsunamigènes sont généralement évoquées dans la littérature pour cet événement majeur. R. OMIRA et al.(2009, 2010) ont modélisé les tsunamis 
générés par chacune de ces sources; À savoir la faute de la Banque Gorringe (GB) [16], la faute de Horseshoe (HS), la faute de Marques de Pombal (MP) [17], la faute de la Banque Portimao (PB), et la faute de Cadiz Wedge (CW) [18] (figure 2). La relation de mise à l'échelle de Scholz (1982) qui repose sur les longueurs de défaut et la relation longueur / largeur a été utilisée pour obtenir l'ampleur du glissement de chaque scénario.
En ce qui concerne le module de cisaillement $(\mu)$ qui caractérise la rigidité de la zone de rupture du tremblement de terre, une valeur de $3 \times 10^{10} \mathrm{~Pa}$ est adoptée pour les cinq failles.

Les paramètres de défaut pour les scénarios étudiés sont illustrés dans le tableau 1. Dans la Figure 2, les scénarios sont tracés.

Tableau 1. Paramètres de fautes typiques des scénarios tsunamigènes candidats dans la région du golfe de Cadix. L: la longueur de la faute en kilomètres; W: la largeur de la faille en kilomètres; D: la profondeur depuis le fond de la mer jusqu'au sommet de la faille en kilomètres; $\mu$ : le module de cisaillement et Mw: l'amplitude du moment.

\begin{tabular}{|c|c|c|c|c|c|c|c|c|c|c|c|c|}
\hline \multirow{2}{*}{$\begin{array}{l}\text { Scenario } \\
\text { name }\end{array}$} & \multirow{2}{*}{$\begin{array}{l}\mathrm{L} \\
(\mathrm{km})\end{array}$} & \multirow{2}{*}{$\begin{array}{l}\text { W } \\
(\mathrm{km})\end{array}$} & \multicolumn{2}{|c|}{ Epicenter coordinates } & \multirow{2}{*}{$\begin{array}{l}\mathrm{D} \\
(\mathrm{km})\end{array}$} & \multirow{2}{*}{$\begin{array}{l}\text { Slip } \\
(\mathrm{m})\end{array}$} & \multirow{2}{*}{$\begin{array}{l}\text { Strike } \\
\left({ }^{\circ}\right)\end{array}$} & \multirow{2}{*}{$\begin{array}{l}\text { Dip } \\
\left({ }^{\circ}\right)\end{array}$} & \multirow{2}{*}{$\begin{array}{l}\text { Rake } \\
\left(^{\circ}\right)\end{array}$} & \multirow{2}{*}{$\begin{array}{l}\mu \\
(\mathrm{Pa})\end{array}$} & \multirow[t]{2}{*}{$\mathrm{Mw}$} & \multirow{2}{*}{$\begin{array}{l}\text { Source } \\
\text { Zone }\end{array}$} \\
\hline & & & Lon & Lat & & & & & & & & \\
\hline GBF & 127 & 60 & -11.332 & 36.665 & 5.0 & 8.3 & 233.0 & 35 & 90 & $3.0 \times 10^{10}$ & 8.1 & GBZ \\
\hline HSF & 165 & 70 & -9.913 & 35.796 & 4.0 & 10.7 & 42.1 & 35 & 90 & $3.0 \times 10^{10}$ & 8.3 & HMZ \\
\hline MPF & 129 & 70 & -9.890 & 36.574 & 4.0 & 8.0 & 20.0 & 35 & 90 & $3.0 \times 10^{10}$ & 8.1 & HMZ \\
\hline PBF & 105 & 55 & -8.585 & 36.314 & 2.5 & 7.2 & 266.3 & 24 & 90 & $3.0 \times 10^{10}$ & 8.0 & $\mathrm{AZ}$ \\
\hline $\mathrm{CWF}$ & 168 & 200 & -8.059 & 35.407 & 5.0 & 10 & 349.0 & 5 & 90 & $3.0 \times 10^{10}$ & 8.6 & $\mathrm{ABZ}$ \\
\hline
\end{tabular}

Les données bathymétriques/ topographiques utilisées
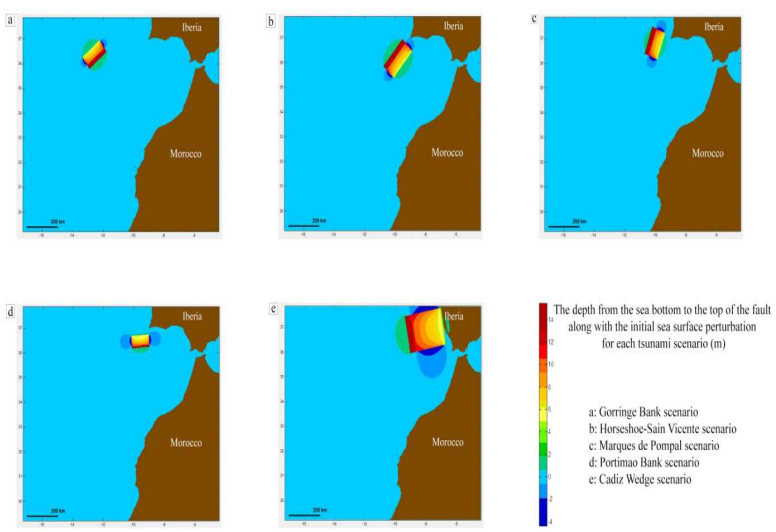

Fig. 2. Perturbation initiale de la surface de la mer pour chaque scénario de tsunami dans le golfe de Cadix; (a) Scénario de la Banque de Gorring, (b) Scénario de Horseshoe; (c) Scénario de Marques de Pombal, (d) Scénario de la Banque de Portimao, (e) Scénario de Cadix Wedge.

\subsection{Modélisation numérique du Tsunami}

Pour simuler la propagation du tsunami, nous utilisons un code non linéaire et un modèle de terrain numérique à haute résolution qui intègre des données d'infrastructures bathymétriques, topographiques et côtières.

Une grille de données bathymétriques / topographiques est générée pour couvrir l'océan atlantique et les côtes marocaines, cette grille a servit comme base pour la réalisation de la modélisation du tsunami.

Le résultat de ces processus a été la génération d'une couche de grille (figure 3), permettant une meilleure représentation des structures bathymétriques, topographiques et côtières afin de mener une modélisation plus réaliste de l'impact du tsunami dans les côtes du Maroc. proviennent de la base de données internationale NOAA, National Oceanic and Atmospheric Administration.

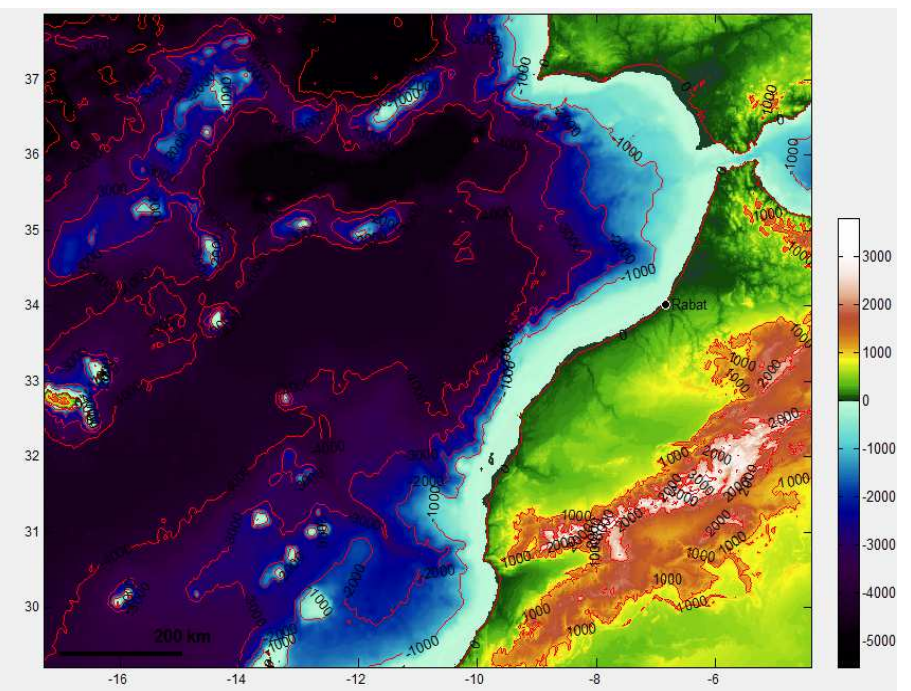

Fig. 3. Modèle d'élévation numérique intégrant les caractéristiques bathymétriques et topographiques de la côte marocaine

\section{Résultats}

La simulation de la propagation des vagues de tsunami a été réalisé dans le but d'analyser leur comportement en s'approchant du littoral afin de comparer les résultats pour tous les scénarios considérés et pouvoir tirer celui qui affecte le plus les côtes marocaines.

\subsection{Scénario Gorringe Bank (GB) Mw = 8.1}

Le scénario $\mathrm{Mw}=8,1 \mathrm{~GB}$, est considéré comme une source candidate pour l'événement Lisbonne de 1755 [16]. La simulation numérique montre une direction de l'énergie du tsunami vers le nord, car la caractéristique bathymétrique de la Gorringe Bank empêche une propagation efficace vers les côtes du golfe de Cadix 
(figure 4). L'analyse de la propagation des vagues de tsunami près du littoral marocain montre des similitudes dans le comportement des vagues avec celles déclenchées par les scénarios HS et MP.

Ces similitudes sont dues à la proximité de ces défauts sismiques ainsi qu'à l'azimut de défaut presque similaire.
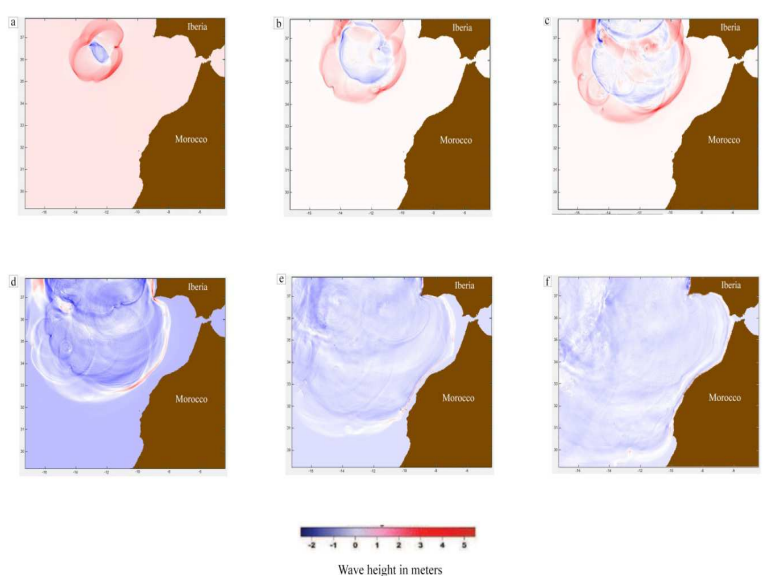

Fig. 4. Simulation de la propagation des vagues du tsunami, depuis la zone épicentrale, suite au scénario Mw = 8.1 GB

\subsection{Scénario Horseshoe (HS) Mw = 8.3}

L'examen des résultats des simulations indique que l'énergie du tsunami du scénario $\mathrm{Mw}=8.3 \mathrm{HS}$ est principalement orientée vers les côtes du nord-ouest du Maroc (figure 5).
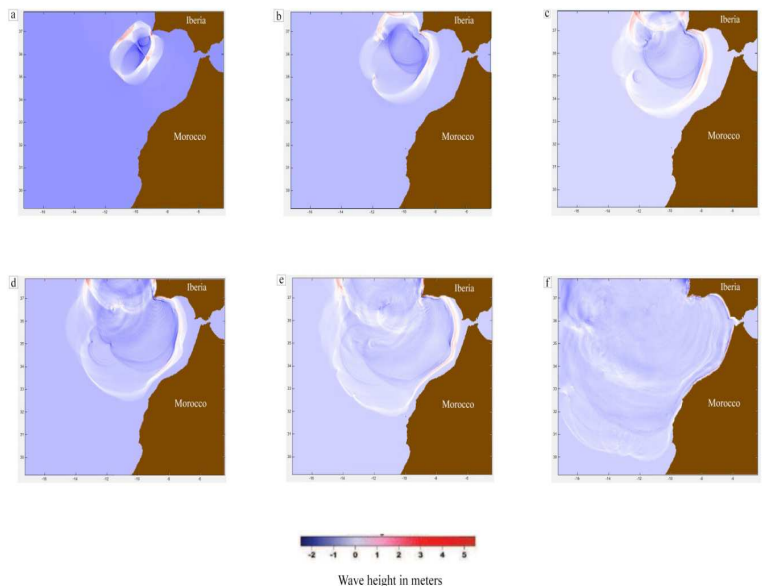

Fig. 5. Simulation de la propagation des vagues du tsunami, depuis la zone épicentrale, suite au scénario Mw $=8.3 \mathrm{HS}$

\subsection{Scénario Marques de Pombal (MP) Mw = 8.1}

Le scénario $\mathrm{Mw}=8.1 \mathrm{MP}$, est basé sur une grande structure tectonique active qui est considérée comme l'une des sources candidates pour l'événement de 1755 [17]. Les simulations numériques montrent que le scénario $\mathrm{Mw}=8.1 \mathrm{MP}$ a déclenché un grand tsunami dans la région du Golfe de Cadix (figure 6). La profondeur de l'eau moins profonde dans la zone hors SW Iberia entraîne une diminution de l'impact du tsunami le long des côtes atlantiques du Maroc.
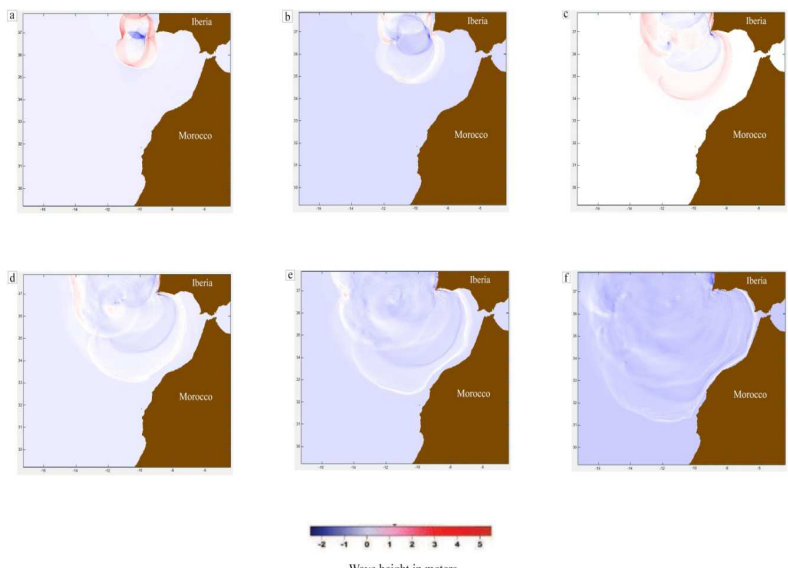

Fig. 6. Simulation de la propagation des vagues du tsunami, depuis la zone épicentrale, suite au scénario $\mathrm{Mw}=8.1 \mathrm{MP}$

\subsection{Scénario Portimao Bank (PB) Mw = 8.0}

Le scénario $\mathrm{Mw}=8.0 \mathrm{~PB}$, par son emplacement très proche du Golfe de Cadix, présente un impact principalement sur cette région.
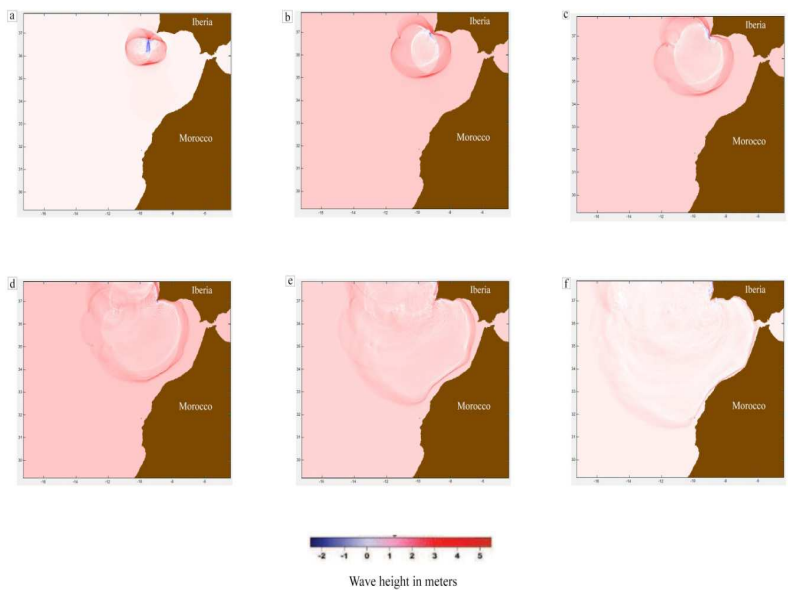

Fig. 7. Simulation de la propagation des vagues du tsunami, depuis la zone épicentrale, suite au scénario $\mathrm{Mw}=8.0 \mathrm{~PB}$

\subsection{Scénario Cadiz Wedge (CW) $M w=8.6$}

La dalle de subduction Cadiz Wedge a été proposée par Gutscher (2004) comme responsable de la génération de l'événement de 1755. La modélisation numérique indique que la localisation et l'azimut du scénario CW contraignent les modèles d'énergie du tsunami et la plupart des énergies sont dirigées vers le détroit de Gibraltar (figure 8). 


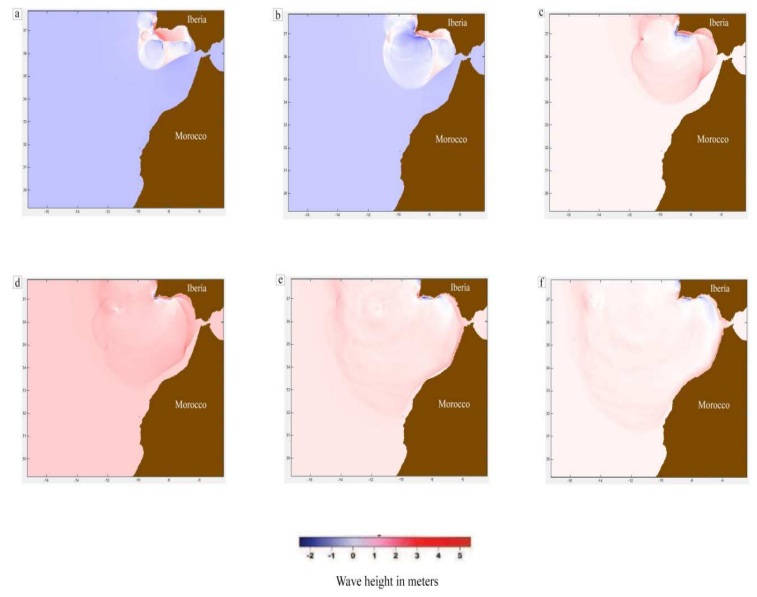

Fig. 8. Simulation de la propagation des vagues du tsunami, depuis la zone épicentrale, suite au scénario $\mathrm{Mw}=8.6 \mathrm{CW}$

\section{Discussion}

Il est clair que la bathymétrie du domaine de propagation, l'emplacement de la faute et son azimut sont les principaux facteurs qui limitent la distribution de l'énergie du tsunami et la direction des hauteurs des ondes. Pour les scénarios HS, MP et GB ayant des sites proches, les propagations du tsunami près des rives marocaines présentent certaines similitudes dans les caractéristiques et la directivité. Concernant le scénario $\mathrm{PB}$, son emplacement et sa directivité induisent une propagation vers la région du Golfe de Cadix. L'exception concerne le scénario $\mathrm{CW}$, pour lequel la propagation du tsunami à proximité de la côte a une directivité différente des autres scénarios considérés. Cela s'explique principalement par l'emplacement de cette source provoquant une propagation du tsunami et des modes d'énergie totalement différents par rapport aux autres sources.

Ce travail montre le haut niveau de destruction que les zones côtières du Maroc, du Portugal et de l'Espagne pourraient ressentir suite à l'apparition de grands événements tsunami régionaux, et donc le besoin de systèmes robustes pour la préparation et l'alerte au tsunami dans la région pour atténuer ce risque.

\section{Conclusions}

La revue des données historiques, instrumentales et géologiques disponibles sur le risque de tsunami au Maroc permettent de conclure que, comme pour les pays voisins, ce risque est réel et que par conséquent des mesures de prévention et de protection des côtes sont nécessaires d'autant plus que les sources les plus dangereuses se trouvent à proximité ce qui ne permet pas un temps de réaction supérieur à une heure. Ceci implique:

- la connaissance du risque qu'encourt une région donnée en fonction de l'aléa et de la vulnérabilité, y compris la délimitation des zones potentiellement exposées au risque et également des endroits les plus vulnérables; pour ce faire, l'étude doit intégrer dans un système SIG de multiples facteurs relatifs à la structure de l'habitat et de l'environnement physique ainsi que des données socio-économiques;

- éviter de nouvelles constructions dans les zones identifiées comme exposées au déferlement des tsunamis pour minimiser les pertes lors de futurs tsunamis; ici, le rôle de l'aménagement du territoire et de son application stricte est de première importance;

- appliquer des normes de construction aux nouveaux bâtiments (résistance au déferlement des vagues, fondations consolidées, etc.) pour minimiser les dommages dus aux tsunamis;

- protéger des tsunamis les constructions et infrastructures existantes dans les zones exposées via des digues ou des murs anti-tsunamis qui, certes, sont des ouvrages très coûteux et peu esthétiques mais qui ont prouvé leur efficacité;

- prendre des précautions spécifiques pour l'installation d'infrastructures nécessaires durant les crises (hôpitaux, pompiers, protection civile, etc.).

$\mathrm{Si}$ la désignation des institutions et des personnes chargées de l'alerte est pratiquement achevée, reste le problème de la gestion de l'alerte au niveau des populations et de la transmission rapide de l'information. Ici, deux solutions existent: d'une part, l'évacuation horizontale qui consiste à se retirer à l'intérieur des terres sur un point haut et, d'autre part, l'évacuation verticale qui réside dans le fait de se réfugier aux étages supérieurs des bâtiments. Cette dernière solution est préconisée lorsque le temps d'évacuation horizontale est très court, lorsque la densité de population est élevée et les moyens d'évacuation horizontale (routes, ponts, etc.).

\section{References}

1. Baptista, M.A. \& Miranda, J.M. (2009). Revision of the Portuguese catalog of tsunamis. Natural Hazards and Earth System Sciences, 9, 25-42.

2. Kaabouben, F., Baptista, M.F., Iben Brahim, A., El Mouraouah, A. \& Toto, A. (2009). On the Moroccan tsunami catalogue. Natural Hazards and Earth System Sciences, 9, 1227-1236.

3. Carmona, P. \& Ruiz, J.M. (2009). Geomorphological evolution of the river Loukkos estuary around the Phoenician city of Lixus on the Atlantic littoral of Morocco. Geoarcheology, 24(6), 821 -845.

4. Gjevik, B., Moe, H., Ommundsen, A., 1997. Sources of the Maelstrom. Nature 388 (28), 837-838

5. Mellas, S., Leone, F., Omira, R., Gherardi, M., Baptista, M.A., Zourarah, B., Péroche, M. \& Lagahé, E. (2012). Le risque tsunamique au Maroc: modélisation et évaluation au moyen d'un premier jeu d'indicateurs d'exposition du littoral atlantique. Physio-Géo, 6, 119-139.

6. El Alami, S.O. \& Tinti, S. (1991). A preliminary evaluation of the tsunami hazards in the Moroccan coasts. Science of Tsunami Hazards, 9(1), 31-38.

7. Elmrabet, T. (2005) The great earthquakes in the Maghreb region and their consequences on man and environment. Imprimerie Beni Snassene, Rabat (en Arabe).

8. Omira, R., Baptista, M.A., Miranda, J.M., Toto, E., Catita, C. \& Catalao, J. (2010). Tsunami vulnerability assessment of Casablanca-Morocco using numerical modeling and GIS tools. Natural Hazards, 54(1), 75-95. 
9. Omira, R., Baptista, M.A., Leone, F., Matias, L., Mellas, S., Zourarah, B., Miranda, J.M., Carrilho, F. \& Cherel, J.P. (2013). Performance of coastal sea-defense infrastructure at El Jadida (Morocco) against tsunami threat: lessons learned from the Japanese 11 March 2011 tsunami. Natural Hazards and Earth System Sciences, 13, 1779-1794.

10. Mellas, S. (2012). Evaluation du risque tsunamique sur le littoral atlantique marocain. Thèse de Doctorat, Université Chouaib Doukkali, Faculté des Sciences d'El Jadida.

11. Ambraseys, N.N. (1962). Data for the investigation of the seismic sea-waves in the Eastern Mediterranean. Bulletin of the Seismological Society of America, 52(4), 895-913.

12. Abe, K. (1979). Quantification of tsunamigenic earthquakes by the Mt scale. Tectonophysics, 166(1), 2734.

13. Mansinha, L., Smylie, D.E., (1971). The displacement fields of inclined faults. Bulletin of the Seismological Society of America. 61 (5): 1433-1440.

14. Okada, Y., (1985). Surface deformation due to shear and tensile faults. In a half-space. Bulletin of the Seismological Society of America. 75 (4): 1135-1154.

15. Gutscher, M. A., Dominguez, S., Graham, K., Westbrook, Leroy, P., (2009). Deep structure, recent deformation and analog modeling of the Gulf of Cadiz accretionary wedge: Implications for the 1755 Lisbon earthquake. Tectonophysics, Elsevier, 475 (1), pp.85-97.

16. Johnston, A., (1996). Seismic moment assessment of earthquakes in stable continental regions - III. New Madrid, 1811-1812, Charleston 1886 and Lisbon 1755. Geophys. J. Int. 126, 314-344.

17. Zitellini, N., et al., (2001). Source of 1755 Lisbon earthquake and tsunami investigated. Eos (Transactions, American Geophysical Union) 82, 285-291.

18. Gutscher, M. A., Dominguez, S., Westbrook, G., Gente, P., Babonneau, N., Mulder, T., Gonthier, E., Bartolome, R., Luis, J., Rosas, F., Terrinha, P., and the Delila and DelSis Scientific Teams. (2008). Tectonic shortening and gravitational spreading in the Gulf of Cadiz accretionary wedge: observations from multi-beam bathymetry and seismic profiling. Journal of Marine and Petroleum Geology, Sp. Vol. on Submarine instabilities. doi:10.1016/j.marpetgeo.2007.11.008.

19. Ammar, A., Mauffret, A., Gorini, C. \& Jabour, H. (2007). The tectonic structure of the Alboran margin of Morocco. Revista de la Sociedad Geologica de España, 20, 247-271.

20. Atillah, A., El Hadani, D., Moudni, H., Lesne, O., Renou, C., Mangin, A. \& Rouffi, F. (2011). Tsunami vulnerability and damage assessment in the coastal area of Rabat and Salé, Morocco. Natural Hazards and Earth System Sciences, 11, 3397-3414.

21. Blanc, P.-L. (2009). Earthquakes and tsunami in November 1755 in Morocco: a different reading of contemporaneous documentary sources. Natural Hazards and Earth System Sciences, 9, 725-738.

22. Debrach, J. (1946). Raz de marée d'origine séismique enregistré sur le littoral atlantique du Maroc. Annales SPGM, 1946, pp. 59-71.

23. Medina, F., Mhammdi, N., Chiguer, A., Akil, M. \& Jaaidi, E.B. (2011). The Rabat and Larache boulder fields; new examples of high-energy deposits related to extreme waves in north-western Morocco. Natural Hazards, 59, 725-747.

24. Mhammdi, N., Medina, F., Kelletat, D., Ahmamou, M. \& Aloussi, L. (2008). Large boulders along the Rabat coast (Morocco); possible emplacement by the November, 1st, 1755 a.d. tsunami. Science of Tsunami Hazards, 27(1), 1730.

25. Mhammdi, N., Medina, F., Trentesaux, A., Font, E., Belkhayat, Z., \& Geawhari, M. A. (2015). Sedimentary evidence of palaeo-tsunami deposits along the Loukkos estuary (Moroccan Atlantic coast). Science of Tsunami Hazards, 34(2), 83-100.

26. Mouzzoun, M., Moustachi, O., Taleb, A., (2012). Evaluation de la vulnérabilité sismique des bâtiments en béton armé par l'utilisation des courbes de fragilités (Seismic vulnerability assessment of reinforced concrete Buildings using fragility curves). J. Mater. Environ. Sci. 3 (6) (2012) 1037-1044. ISSN:2028-2508 CODEN:JMESCN. 